\title{
NCI CTEP SDC Lung, Mediastinal and Pleural Neoplasm Category Terminology
}

National Cancer Institute

\section{Source}

National Cancer Institute. NCI CTEP SDC Lung, Mediastinal and Pleural Neoplasm

Category Terminology. NCI Thesaurus. Code C103023.

Lung, Mediastinal and Pleural Neoplasm is an NCl Cancer Therapeutic Evaluation Program (CTEP) Simplified Disease Classification (SDC) category used to organize cancer-related disease coding that harmonizes with and supports reporting based on the global standard Medical Dictionary for Drug Regulatory Reporting (MedDRA) terminology. 